\title{
THRESHOLD AND CONTINUUM STRUCTURES IN EXOTIC NUCLEI
}

\author{
I.J. THOMPSON, B.V. DANILIN, J. MORTIMER, \\ T. TARUTINA ${ }^{\dagger}$ AND J.A. TOSTEVIN \\ Department of Physics, University of Surrey, Guildford GU2 7XH, U.K.
}

\begin{abstract}
The role of continuum states in nuclei is considered, along with use of breakup theories of stripping and diffractive dissociation in probing nuclear structure. In the breakup of three-body nuclei such as Borromean halo systems, both three-body and two-body continuum final states need to be modelled and measured.
\end{abstract}

\section{Few- and many-body dynamics}

Nuclei typically show few-body behaviour just below and above the cluster separation thresholds. Many exotic nuclei have just one or a few bound states, and show pronounced cluster dynamics even in their ground states. Probing more details of these structures may follow from elastic scattering or cluster transfers, but breakup cross sections are typically the largest. This makes breakup experiments particularly attractive for spectroscopy with weak beams of exotic nuclei.

Breakup mechanisms lead to continuum states, and the continuum has many roles in nuclear structure physics. It appears in several ways, as (a) part of the expansion of bound states; e.g. in RPA for weakly bound states; or (b) dominated by resonances, as 'unbound states' to be identified e.g. with shell model eigenstates above threshold; or (c) in non-resonant continuum; e.g. in breakup reactions. All of these features are important parts of nuclear structure.

I therefore review the structure information that is present in breakup amplitudes, as well as bound structure details that can be extracted from different classes of breakup reactions.

*Permanent address: Russian Research Center "The Kurchatov Institute", 123182 Moscow, Russia

${ }^{\dagger}$ Current address: Departamento de Física Nuclear, Instituto de Física da Universidade de São Paulo, Caixa postal 66318, 05315-970 São Paulo, Brazil 


\section{Knockout to bound final states}

Stripping processes (inelastic breakup) proceed by the removal of a surface nucleon in a high-energy interaction with a target, and can reveal the spectroscopic factors for a wide range of final states. These states may be identified by measurement of coincident $\gamma$-rays.

The eikonal reaction theory for breakup by the removal of one or two neutrons or protons is now extensively recognised $[1,2]$ as a useful tool in probing single-particle structures. Contributions from both stripping and diffractive dissociation need to be calculated from the eikonal theory, which gives the integrated cross sections for these two removal mechanisms.

Recent applications of such analyses have been to

- The ${ }^{9} \mathrm{Be}\left({ }^{17} \mathrm{C},{ }^{16} \mathrm{C} \gamma\right)$ reaction at $60 \mathrm{MeV} / \mathrm{u}[3]$, where $\gamma$-coincidences allow the extraction of spectroscopic factors for the first three levels in ${ }^{16} \mathrm{C}$. Shell-model calculations predict a very small parentage of the ${ }^{16} \mathrm{C}\left(0^{+}\right)$in the ${ }^{17} \mathrm{C}\left(3 / 2^{-}\right)$gs, while a $20 \%$ branch into ${ }^{16} \mathrm{C}\left(0^{+}\right)$ was measured: this may indicate a need for higher order processes in an improved reaction theory.

- The $N=8$ neutron shell closure in ${ }^{12}$ Be has been examined [4], by measuring the production of different ${ }^{11} \mathrm{Be}$ final states after oneneutron stripping.

- The ground state structure of ${ }^{8} \mathrm{~B}$ has been examined by proton removal from ${ }^{8} \mathrm{~B}$ measured at the GSI [5] with $\gamma$ coincidences, where a $15 \%$ branch is seen from the excited ${ }^{7} \mathrm{Be}\left(1 / 2^{-}\right)$core component in the ${ }^{8} \mathrm{~B}$ wave function.

Systematic reviews $[6,1]$ of the extracted spectroscopic factors show values that are significantly less than shell-model values (with a 'reduction factor' $R \sim 0.6$ ) for deeply bound states. The results are in agreement with those from $\left(e, e^{\prime} p\right)$ electron knockout reactions on stable targets, and are believed to reveal correlations from mechanisms not included in the shell model effective interactions, and which couple to configurations outside the assumed model space. These include the short-range correlations from the nucleon-nucleon repulsive core, tensor correlations, and clustering effects. This explanation is supported by noting that the reduction factor is nearer to unity $(R \sim 0.9)$ for weakly-bound initial states, such as those in ${ }^{8} \mathrm{~B},{ }^{9} \mathrm{C}$ and ${ }^{15} \mathrm{C}$. 

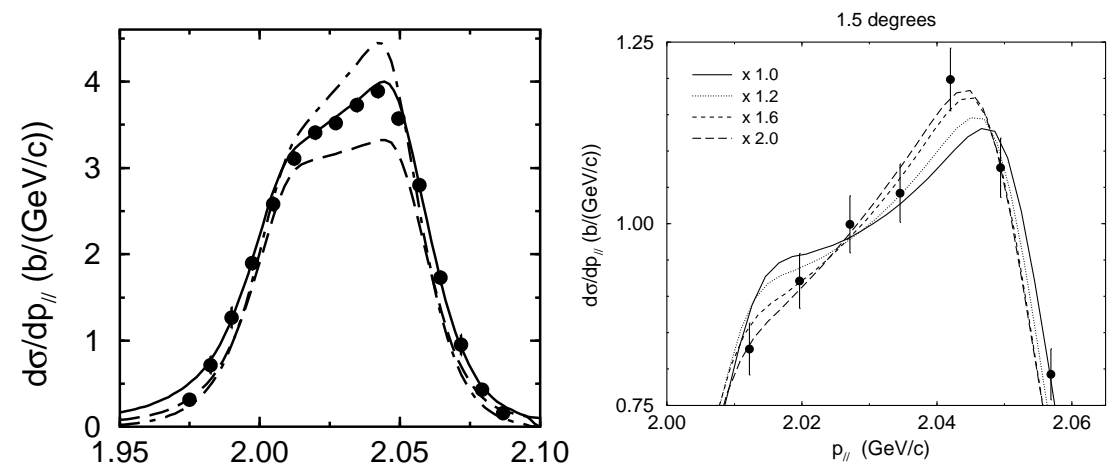

Figure $1 .{ }^{8} \mathrm{~B}+{ }^{208} \mathrm{~Pb} \rightarrow{ }^{7} \mathrm{Be}$ parallel momentum distributions at $44 \mathrm{MeV} / \mathrm{u}$. (Left) with a $3.5^{\circ}$ transverse aperture. Dot-dashed: semiclassical Coulomb; solid: Coulomb+nuclear DWBA; dashed: CDCC coupled channels cross sections. (Right) CDCC calculations with scaled E2 amplitudes, for the same reaction with a $1.5^{\circ}$ aperture.

\section{Diffractive dissociation to unbound two-body states}

Diffraction dissociation (elastic breakup), in which the projectile is dissociated by elastic interactions of the projectile fragments with the target remaining in its ground state, probes continuum excited states. For nuclei near the dripline, with few discrete states, these breakup reactions are the principal probe of excited states.

To measure the E1 and E2 multipole breakup of ${ }^{8} \mathrm{~B}$, for example, we need spectroscopy of the non-resonant continuum. This nucleus has a oneproton bound state at $-0.137 \mathrm{MeV}$ with dominant structure $\left[{ }^{7} \mathrm{Be}\left(3 / 2^{-}\right) \otimes\right.$ $\left.\left(0 p_{3 / 2}+0 p_{1 / 2}\right)\right]_{2^{+}}$, as reported above. To find the $\mathrm{B}(\mathrm{E} 1)$ and $\mathrm{B}(\mathrm{E} 2)$ distributions, the transitions $p \rightarrow s, d$ need to be accurately known. These E1 and E2 amplitudes interfere in the $p_{\|}\left({ }^{7} \mathrm{Be}\right)$ parallel momentum distribution, giving a forward-backward asymmetry [7].

The accurate calculation of these asymmetries therefore requires full treatment of possible quantal effects, nuclear+Coulomb interference, and higher-order processes [8]. It is known that higher-order $(\mathrm{E} 1)^{2} * \mathrm{E} 2$ interference reduces the observed asymmetries, as shown by the all-order CDCC calculation (dashed curve) in the left part of Fig. 1.

The puzzle that now arises is that the higher-order effects reduce the calculated asymmetry to less than is manifest by the data of [7], requiring, as shown in right part of Fig. 1, that the E2 amplitudes be increased to fit the data [8]. There is a need for further work in structure models to see whether these properties of the non-resonant breakup continuum 
may be microscopically reproduced, or whether the ${ }^{7} \mathrm{Be}\left(1 / 2^{-}\right)$excited core component needs to be included in the breakup mechanism.
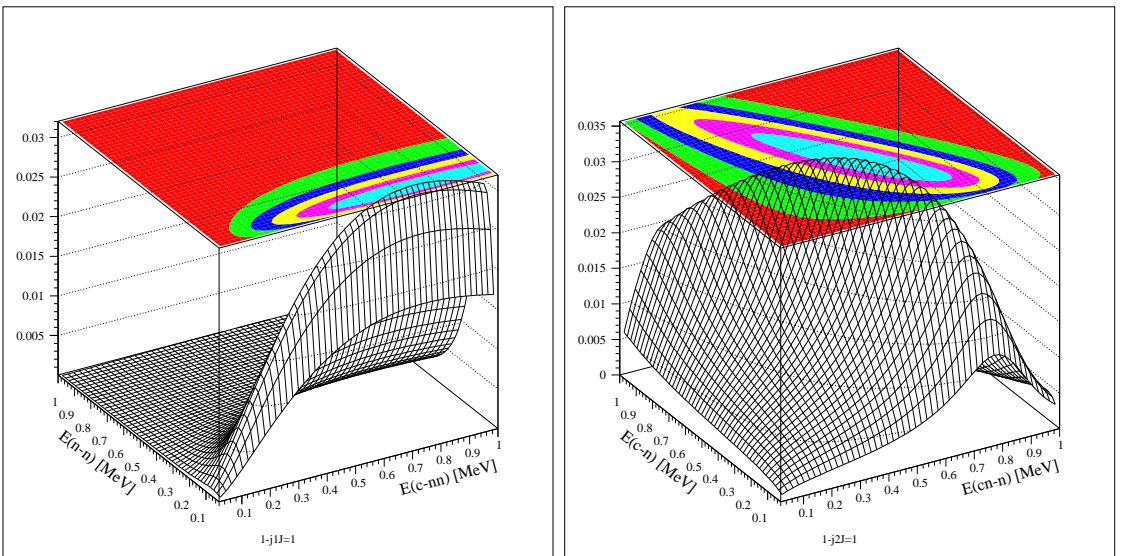

Figure 2. Three-body scattering wave functions averaged over angles, as functions of two Jacobi energies. (Left) Effect of the n-n virtual state. (Right) Effect of hypothetical n-n higher energy resonance.

\section{Diffractive dissociation to unbound three-body states}

Similar questions arise in the breakup of three-body systems, where now the distinctions between resonant and non-resonant continua become more complicated, and more interesting. In probing the three-body continuum of Borromean systems (those having no bound binary subsystems), possible experimental signatures may be the energy correlations in the continuum scattering.

Using the continuum states of ${ }^{6} \mathrm{He}$ as an example, we plot in Fig. 2 the scattering wave functions averaged over the angles of the relative motion. The left part shows the correlations obtained from the n-n virtual state, with a strong amplification at low $E_{n n}$ values that is independent of $E_{c-n n}$. The right part shows the effect of a hypothetical n-n resonance at higher energy in $E_{c-n}, E_{c n-n}$ energy coordinates, where there is now a uniform distribution between these energies.

We now calculate these correlation plots using our three-body models of excited ${ }^{6} \mathrm{He}$ continuum states [9]. The left part of Fig. 3 shows the well known $2^{+}$resonance at $1.8 \mathrm{MeV}(0.9 \mathrm{MeV}$ above the breakup threshold), showing clear resonant energy distributions across both the Jacobi energy 
variables shown. In the right part, by contrast, we plot the results of our continuum calculations for dipole $1^{-}$states. Here, there are no clear resonant peaks, either in individual or in summed energy variables. The dipole response is amplified because of attractive dynamical correlations, but not sufficiently to produce a recognisable resonance.
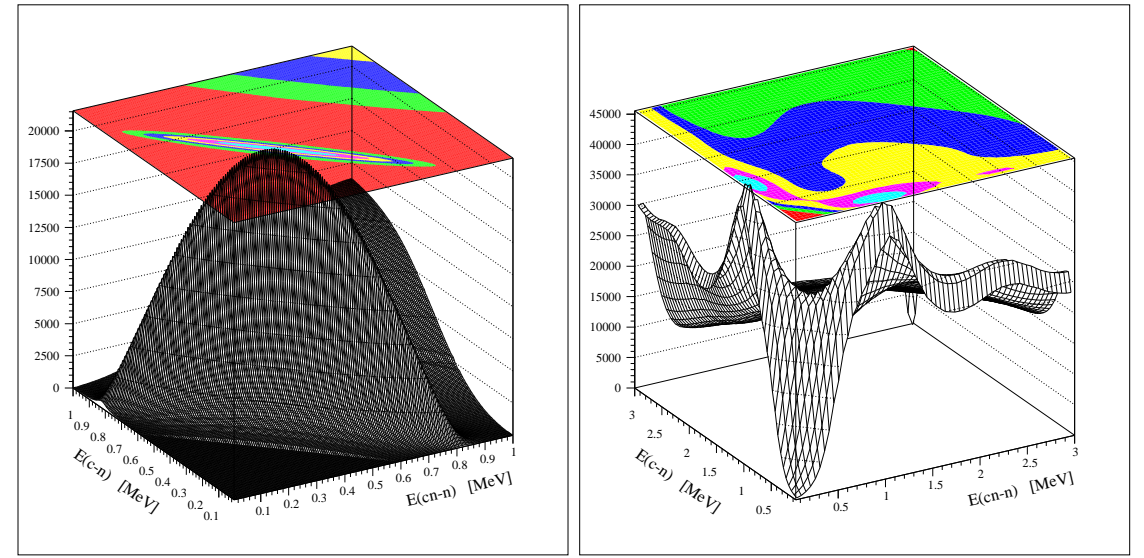

Figure 3. Energy correlation plots (as in Fig. 2) of excited ${ }^{6} \mathrm{He}$ continuum states. (Left) The well known $2^{+}$resonance at $1.8 \mathrm{MeV}$. (Right) Dipole $1^{-}$states.
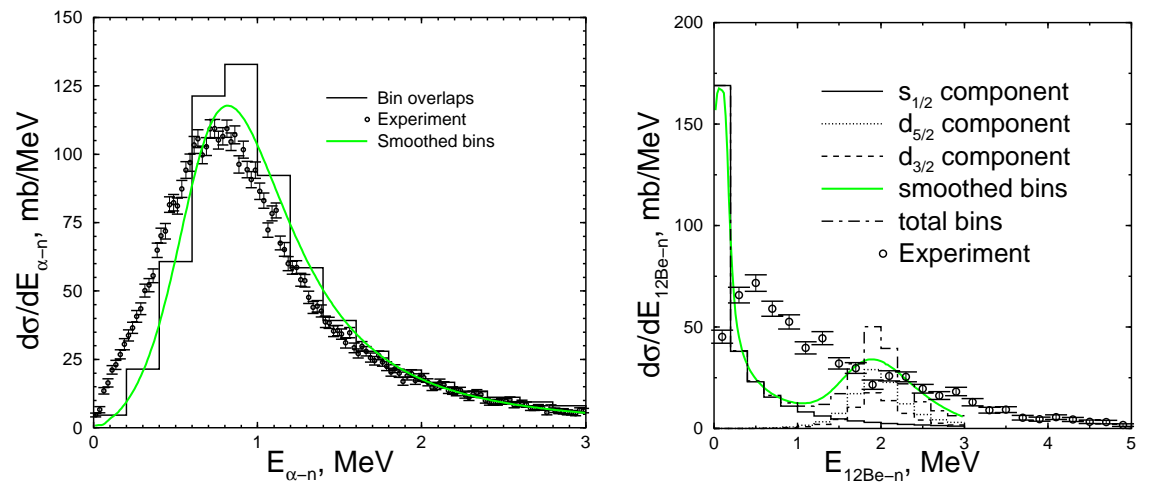

Figure 4. (Left) Nucleon removal cross sections at $240 \mathrm{MeV} / \mathrm{u}$ to ${ }^{5} \mathrm{He}^{*}$ from the ${ }^{6} \mathrm{He}$ three-body ground state. The data are from [10]. (Right) to ${ }^{13} \mathrm{Be}^{*}$ from the ${ }^{14} \mathrm{Be}$ g.s. The data are from [13]. 


\section{Knockout producing unbound subsystems}

There is also the possibility of probing continuum states of Borromean subsystems by removal of one nucleon. The removal of a neutron from ${ }^{6} \mathrm{He}$, ${ }^{11} \mathrm{Li}$ or ${ }^{14} \mathrm{Be}$, for example, populates states of ${ }^{5} \mathrm{He},{ }^{10} \mathrm{Li}$ or ${ }^{13} \mathrm{Be}$, respectively.

Experiments have measured the decay spectra of ${ }^{5} \mathrm{He}\left(={ }^{4} \mathrm{He}+\mathrm{n}\right)$, ${ }^{13} \mathrm{Be}\left(={ }^{12} \mathrm{Be}+\mathrm{n}\right)$, etc., so we calculate these energy correlations within eikonal reaction theory, using good 2-body and 3-body structure models of the $\mathrm{A}+1$ or the $\mathrm{A}+2$ nuclei, respectively. For the ${ }^{6} \mathrm{He}$ case, we first calculate the overlaps $\left\langle{ }^{5} \mathrm{He}\left(E_{\alpha-n}\right)\right|{ }^{6} \mathrm{He}$ gs $\rangle$ for a range of ${ }^{5} \mathrm{He}\left(E_{\alpha-n}\right)$ bin states, then smooth the histogram of the calculated eikonal cross sections with the experimental energy resolutions. In the left part of Fig. 4 we compare theoretical predictions with the GSI data [10]. The total nucleon removal cross sections are calculated [11] to be $\sigma_{\text {str }}=137 \mathrm{mb}$ and $\sigma_{\text {diff }}=38$ $\mathrm{mb}$ which compare very favourably with the measured values of $127 \pm 14 \mathrm{mb}$ and $30 \pm 5 \mathrm{mb}$ respectively.

These good agreements lead us to try the method for the ${ }^{14} \mathrm{Be} \rightarrow{ }^{13} \mathrm{Be}$ knockout reaction. In ${ }^{13} \mathrm{Be}$, initial models [12] require a low-lying $s$-wave virtual state alongside a $d$-wave resonance at $\sim 2 \mathrm{MeV}$. The integrated cross sections obtained with such a model are $\sigma_{\text {str }}=109 \mathrm{mb}$ and $\sigma_{\text {diff }}=109$ $\mathrm{mb}$, compared with the measured values [13] of $125 \pm 19 \mathrm{mb}$ and $55 \pm 19 \mathrm{mb}$ respectively, and the energy spectrum is shown in right part of Fig. 4. For the nucleon removal from this nucleus, we see that the experimental energy spectrum is smoother than that predicted theoretically, with no pronounced virtual-s and resonant- $d$ state peaks. More recent models [11] with core excitation are expected to predict similar energy distributions. We conclude that either there are further sources of experimental energy smoothing, or that the simplified eikonal reaction mechanism is not adequate, which assumes that the produced ${ }^{13} \mathrm{Be}^{*}$ unbound states are spectators and do not undergo mixing and energy sharing during the reaction.

\section{Conclusions}

Near-threshold states give rise to cluster dynamics and strong coupling to breakup channels. Many of the new, exotic nuclei near the driplines have only one bound state. The modelling and measurement of continuum states, as well as of the mechanisms which produce them, are therefore necessary in order to probe spectroscopic structures in the new era of nuclear structure physics. Spectroscopy of states in the continuum is as important as the spectroscopy of discrete states, whether those are bound states or discrete 
resonances.

\section{Acknowledgments}

This work was supported by EPSRC grants GR/R25514 and GR/M82141 (U.K.). One of the authors, T.T., would like to acknowledge partial support of FAPESP, Brazil.

\section{References}

1. P.G. Hansen and J.A.Tostevin, Ann. Rev. Nucl. Part. Sci 53, 219 (2003).

2. J. Tostevin, proceedings of RNB6.

3. V. Maddalena et al., Phys Rev C 63, 024613 (2001).

4. A. Navin et al., Phys. Rev. Lett. 85, 266 (2000).

5. D. Cortina-Gil et al., Phys Lett B 529, 36 (2002), Nucl. Phys. A720, 3 (2003).

6. B.A. Brown et al. Phys Rev C 65, 061601(R) (2002)

7. B. Davids et al., Phys. Rev. Lett. 86, 2750 (2001)

8. J. Mortimer et al., Phys Rev C 65, 64619 (2002).

9. B.V. Danilin et al, Nucl. Phys. A632, 383 (1998).

10. D. Alexandrov et al, Nucl. Phys. A 633, 234 (1998).

11. T. Tarutina, Ph.D. thesis, University of Surrey (Sept 2001).

12. I.J. Thompson and M.V. Zhukov, Phys. Rev. C53, 708 (1996)

13. H. Simon, private communication 